\title{
Herleitung von Grundvorstellungen als normative Leitlinien - Beschreibung eines theoriebasierten Verfahrensrahmens
}

\author{
Alexander Salle (iD) Tomma Clüver
}

Eingegangen: 28. April 2020 / Angenommen: 22. März 2021 / Online publiziert: 16. April 2021

(C) Der/die Autor(en) 2021

Zusammenfassung Grundvorstellungen stellen Richtlinien für die Gestaltung von Lernprozessen sowie für eine strukturierte Erforschung mentaler Repräsentationen bereit. Wie jedoch die Herleitung von Grundvorstellungen vorgenommen werden kann, wird in der Literatur unterschiedlich beschrieben oder nur teilweise expliziert. Im vorliegenden Beitrag wird ein Verfahrensrahmen für die Herleitung von Grundvorstellungen als normative Leitlinien vorgeschlagen. Dieser umfasst fünf Schritte: (1) Festlegung zentraler Konzepte, zu denen Grundvorstellungen formuliert werden sollen, sowie weiterer Richtlinien für den Herleitungsprozess, (2) sachanalytisch begründete Klassenbildung auf Basis relevanter mathematischer Definitionen, der Begriffsphänomenologie sowie empirischer Ergebnisse, (3) Formulierung konkreter Grundvorstellungen anhand der gebildeten Klassen und Analyse ihrer Beziehungen untereinander, (4) Bestimmung von Grundkenntnissen und Analyse der Beziehungen zu anderen Grundvorstellungen, (5) Klärung der didaktischen Relevanz.

Die Herleitung von Grundvorstellungen mit diesem Verfahrensrahmen wird anhand des Sinus- und Bruchzahlbegriffs exemplifiziert.

Schlüsselwörter Grundvorstellungen · Stoffdidaktik · Phänomenologie · Sachanalyse $\cdot$ Sinus 


\title{
Deriving Basic Ideas as Prescriptive Guidelines-Description of a Theory-Based Framework
}

\begin{abstract}
Basic ideas (Grundvorstellungen) are guidelines for the conception of learning processes. They also structure empirical research aiming at analyzing individual mental representations. However, it is often not exactly described how content-specific formulations of basic ideas are carried out.

In this paper, we propose a framework for deriving basic ideas as prescriptive guidelines. It comprises five steps: (1) selection of the central mathematical concepts for which basic ideas should be formulated and a preliminary determination of further guidelines, (2) class formation based on subject-matter analyses of relevant definitions, their phenomenology and empirical findings, (3) formulation of basic ideas based on the previously formed classes and analyses of the relationships between the formulated basic ideas (4) specification of the relevant basic knowledge and analyses of further basic ideas, (5) assessment of the didactical relevance.

The derivation of basic ideas with the proposed framework is carried out exemplarily for the concepts of sine and fractional numbers.
\end{abstract}

Keywords Basic mental representations · Grundvorstellungen - Subject-matter didactics $\cdot$ Phenomenology $\cdot$ Sine

\section{Einleitung}

Welche „Vorstellungen“, „Anschauungen“ und „Verinnerlichungen“ Schülerinnen und Schüler mit mathematischen Begriffen verbinden, beschäftigt seit langer Zeit Mathematikdidaktik, Pädagogik und Psychologie (vom Hofe 1995; vom Hofe und Blum 2016). Im Kern der Forschungsvorhaben steht die Motivation, das Lernen mathematischer Konzepte genauer zu verstehen. Ziel ist es, Verständnisschwierigkeiten durch die Ausbildung adäquater Vorstellungen zu begegnen und die Begriffsbildung zu unterstützen (z.B. Bender 1991; Prediger 2008). Anknüpfend an die genannten Begriffe arbeitet vom Hofe (1995) die Herkunft des Begriffs „Grundvorstellung“ auf; Grundvorstellungen stehen für inhaltliche Deutungen mathematischer Begriffe und gelten als „Elemente der Vermittlung [...] zwischen der Welt der Mathematik und der individuellen Begriffswelt des Lernenden“" (vom Hofe 1995, S. 98; s. auch Greefrath et al. 2016a).

Seit dieser Begriffslegung hat das Grundvorstellungskonzept eine enorme Popularität und Ausgestaltung erfahren. Zum einen konnten in einer Vielzahl empirischer Studien Leistungsunterschiede von Schülerinnen und Schüler mithilfe des Grundvorstellungskonzeptes erklärt werden (z.B. Prediger 2008; vom Hofe et al. 2005; Wartha 2007).

Zum anderen spielen Grundvorstellungen auf schulpraktischer Ebene eine wichtige Rolle: In Bildungsstandards und Lehrplänen findet das Grundvorstellungskonzept explizite Berücksichtigung (z.B. Bildungsstandards für die Allg. Hochschulreife, Ständige Konferenz der Kultusminister der Länder in der Bundesrepublik Deutschland 2015; Nds. Kerncurriculum für das Gymnasium - gymn. Oberstufe, 
Niedersächsisches Kultusministerium 2018). Auch Schulbücher und fachdidaktische Grundlagenwerke greifen Grundvorstellungen als Leitlinien für den Mathematikunterricht auf (z. B. Greefrath et al. 2016a; Padberg und Wartha 2017; vom Hofe et al. 2014).

Zentraler Ausgangspunkt für die genannten Rezeptionen ist die Konkretisierung von Grundvorstellungen in verschiedenen Inhaltsbereichen (Prediger 2010). Dabei wird die Herleitung der Grundvorstellungen in unterschiedlicher Weise und Ausführlichkeit dargestellt: Grundvorstellungen werden entweder anhand der grundlegenden mathematischen Objekte, ihrer Definitionen sowie relevanter Anwendungskontexte hergeleitet (z.B. Greefrath et al. 2016a; Weber 2016), mit Verweis auf fach- und stoffdidaktische Beiträge formuliert (z. B. Hafner 2012; Stölting 2008) oder - oftmals im Rahmen unterrichtspraktischer Beiträge - ohne weitere Explikation der vorgenommenen Herleitung angegeben (z. B. Malle 2003; Salle und Frohn 2017). Welche konkreten Überlegungen bei der Konstruktion der jeweiligen Grundvorstellungen ausgeführt wurden, bleibt häufig implizit. Bisher existiert kein explizierter Rahmen, in dem nötige bzw. mögliche Schritte für eine Herleitung von Grundvorstellungen dargelegt werden.

Basierend auf der bisherigen Forschung zu Grundvorstellungen wird in diesem Artikel ein solcher theoretisch fundierter Verfahrensrahmen vorgeschlagen. Dieses Rahmenmodell kann einen wichtigen Beitrag für die Herleitung neuer Grundvorstellungen zu mathematischen Begriffen leisten. Weiterhin können anhand dieses Rahmens die Herleitungsprozesse bereits formulierter Grundvorstellungen expliziert und nachvollzogen werden. Der vorliegende Beitrag verfolgt somit vor allem ein methodologisches Anliegen. Die in diesem Beitrag vorgestellten Schritte stellen keinen „Algorithmus“ zur Herleitung von Grundvorstellungen dar (vgl. Bender 1991, S. 56); vielmehr werden essenzielle Begriffe geklärt und Schritte angegeben, die für eine Herleitung von Grundvorstellungen grundlegend sind.

Nach der Darstellung des Grundvorstellungskonzeptes (Abschn. 2) wird basierend auf Überlegungen zu stoffdidaktisch orientierten Sachanalysen, zur Phänomenologie mathematischer Begriffe sowie zu bisher formulierten Grundvorstellungen (Abschn. 3) ein Verfahrensrahmen für die Herleitung von Grundvorstellungen vorgeschlagen (Abschn. 4). Dieses Verfahren wird exemplarisch für ausgewählte Grundvorstellungen zum Sinus- und Bruchzahlbegriff dargestellt (Abschn. 5). Abschließend werden Perspektiven des Herleitungsverfahrens aufgezeigt (Abschn. 6).

\section{Grundvorstellungen mathematischer Inhalte}

\subsection{Begriffsdefinition}

Grundvorstellungen konzeptualisieren „,idealtypische mentale Repräsentationen“ (Griesel et al. 2019, S. 129) mathematischer Objekte und Sachverhalte und werden somit zunächst als stofflich basierte Leitlinien für Lernprozesse formuliert. Mentale Repräsentationen werden kognitionspsychologisch als ,geistige Abb[ildungen] von Wahrnehmung, Gedächtnisinhalten o[der] Denkprozessen“ (Städtler 2003, S. 676) spezifiziert. In Bezug auf das Grundvorstellungskonzept sind diese geistigen 
Abbildungen nicht ausschließlich bildhaft $\mathrm{zu}$ verstehen; vielmehr sind enaktive (z.B. Addition repräsentiert als Zusammenfassen oder Hinzufügen von Elementen, Hasemann und Gasteiger 2014), ikonische (z. B. Integral repräsentiert als der Flächeninhalt zwischen Graph und x-Achse, Greefrath et al. 2016a) oder symbolische (z.B. Logarithmus repräsentiert als die um eins verminderte Stellenzahl einer Zahl, Weber 2013) Repräsentationsformate „denkbar“ (Bruner 1964; Lorenz 2017).

Das „Idealtypische“ der mentalen Repräsentationen bezieht sich bei Grundvorstellungen, die an der Schnittstelle von Mathematik und Realität verortet sind, sowohl auf Strukturübereinstimmungen zwischen den Repräsentationen und dem betrachteten mathematischen Konstrukt als auch auf Strukturübereinstimmungen zwischen den Repräsentationen und dem betrachteten realitätsnahen Sachverhalt (Griesel et al. 2019; Kleine et al. 2005). Im Sinne des Verkürzungsmerkmals von Modellen nach Stachowiak (1973) weisen die idealtypischen Repräsentationen in beiden Fällen hinreichend strukturelle Übereinstimmungen auf und sind nicht als vollständig strukturgleich $\mathrm{zu}$ verstehen. ${ }^{1}$

Grundvorstellungen erfüllen drei Eigenschaften: Die erste Eigenschaft betrifft eine den Grundvorstellungen inhärente inhaltliche Deutung mathematischer Begriffe (Greefrath et al. 2016a, 2016b; vom Hofe 1995). Grundvorstellungen leisten somit einen Beitrag zu einem sinnstiftenden Mathematiktreiben, indem sie auf Anwendungen der Begriffe, auf die historische Begriffsgenese und auf fundamentale Ideen Bezug nehmen (Leuders et al. 2011; Vohns 2005). Insbesondere sollen Grundvorstellungen den Lernenden eine Sinnkonstituierung der Begriffe durch eine entsprechende Anknüpfung an deren Vorwissen ermöglichen (vom Hofe 1995). Dabei können sich diese Erfahrungen sowohl auf die unmittelbare Lebenswelt der Lernenden (sog. primäre Grundvorstellungen) als auch auf gedankliche Operationen mit mathematischen Darstellungsmitteln (sog. sekundäre Grundvorstellungen) beziehen (vom Hofe und Blum 2016).

Die Adäquatheit der inhaltlichen Deutungen ist vor dem Hintergrund des Gültigkeitsbereichs des betrachteten mathematischen Begriffs und des Bezugsrahmens der Grundvorstellung charakterisiert (s. auch Reinhold 2019; Roos 2020). Der Gültigkeitsbereich eines Begriffs gibt dessen (mathematische) Reichweite an. Beispielsweise kann die Multiplikation im Gültigkeitsbereich der natürlichen Zahlen adäquat als wiederholte Addition des Multiplikanden gedeutet werden, in den rationalen Zahlen jedoch nur noch in Sonderfällen (Padberg und Wartha 2017). Weitet man den Gültigkeitsbereich eines Begriffs aus, so können Anpassungen bzw. Erweiterungen von Grundvorstellungen und deren Bezugsrahmens erforderlich sein. Die erforderlichen Anpassungen werden als Grundvorstellungsumbrüche beschrieben (Padberg und Wartha 2017; Wartha 2007; s. auch Abschn. 2.3). Im Bezugsrahmen einer Grundvorstellung werden diejenigen Kenntnisse, Kompetenzen und (Grund-)Vorstellungen zusammengefasst, die notwendig für den Aufbau dieser Grundvorstellungen sind. Die Kenntnis der Addition natürlicher Zahlen ist damit beispielsweise im Bezugsrahmen der oben betrachteten Grundvorstellung zur Multiplikation verortet.

\footnotetext{
1 Welche Übereinstimmungen in den konkreten Fällen hinreichend sind, wird dabei in erster Linie vom Forschenden entschieden. Dieser Umstand wird im vorgestellten Verfahren (s. Abschn. 4) expliziert.
} 
Das in der ersten Eigenschaft des Grundvorstellungskonzeptes verankerte Ziel, einem bedeutungslosen Mathematiktreiben durch eine Anknüpfung der mathematischen Konzepte an die Vorerfahrungen der Lernenden entgegenzuwirken, ist ebenfalls Ausgangsgedanke der Prototypen nach Dörfler (1990) und der Kernideen nach Gallin und Ruf (1993). Verglichen mit Grundvorstellungen sind die Ausrichtungen der Konzepte jedoch unterschiedlich: Um „,bedeutungskonstitutive Handlungen“ mit dem Begriff zu ermöglichen, hebt Dörfler (1990, S. 104) die Entwicklung schematisierter Vorstellungen, sogenannter Prototypen, hervor und betont damit „die Rekonstruktion und Abstraktion der zentralen und ,definierenden' Eigenschaften und Beziehungen des entsprechenden Begriffs". Mit dem Konzept der Kernideen wird die Bedeutungskonstruktion bei der Unterrichtsplanung herausgestellt (Gallin und Ruf 1993). Im Rahmen des dialogischen Lernmodells werden hierbei nicht nur die Sichtweisen und Erfahrungen der Lernenden, sondern insbesondere auch die der Lehrperson bedacht (Ruf 2008).

Neben der Sinnkonstituierung mathematischer Begriffe werden zwei weitere Kerngedanken in der Grundvorstellungsidee fokussiert: Die zweite Eigenschaft von Grundvorstellungen drückt aus, dass sie über die Anknüpfung an verschiedene Handlungen und Operationen ein ,operatives Handeln auf der Vorstellungsebene“ (vom Hofe 1995, S. 98) ermöglichen sollen. Über die „Verinnerlichung“ von Handlungen im Piagetschen Sinne sollen die Lernenden befähigt werden, flexibel mit gedanklichen Konstrukten zu arbeiten. Dies steht in enger Verbindung mit dem beweglichen Denken nach Roth (2005, S. 38), bei dem es ebenfalls um „das bewusste, aktive Verändern einer evtl. zunächst statischen Konfiguration einschließlich der Reflexion der Konsequenzen dieser Veränderung" geht.

Die dritte Eigenschaft von Grundvorstellungen bezieht sich auf ihre zentrale Rolle bei der Anwendung mathematischer Begriffe. Durch die sich in Grundvorstellungen widerspiegelnde inhaltliche Deutung mathematischer Begriffe sowie deren Anknüpfung an bekannte Sach- und Handlungssituationen nehmen sie eine Vermittlungsfunktion bei Übersetzungsprozessen ein (Stölting 2008; vom Hofe und Blum 2016). Dies zeigt sich im Modellierungskreislauf bei primären Grundvorstellungen vorrangig in ihrer Bedeutung beim Mathematisieren eines realen Modells bzw. beim Interpretieren der mathematischen Ergebnisse in einem Sachzusammenhang; beim Übersetzen von einer Darstellung in eine andere können vor allem sekundäre Grundvorstellungen genutzt werden.

Um Grundvorstellungen in Übersetzungsprozessen nutzbar zu machen, sollte zudem auf bestimmte Wissensbestände bei Lernenden zurückgegriffen werden können (Stölting 2008). Diese sogenannten Grundkenntnisse umfassen „Kenntnisse zu Auswirkungen von Eigenschaften mathematischer Konzepte bei Übersetzungsprozessen“ (Stölting 2008, S. 39). Grundkenntnisse können jedoch nicht nur Voraussetzung für eine Verwendung von Grundvorstellungen sein, sondern auch ,,als konkrete Ausprägungen von Grundvorstellungen aufgefasst werden“ (Stölting 2008, S. 39); in diesem Fall ergeben sich die Grundkenntnisse aus der Arbeit mit Grundvorstellungen. 


\subsection{Normativer, deskriptiver und konstruktiver Aspekt}

Die bereits beschriebene Bedeutung von Grundvorstellungen für Fachdidaktik und Mathematikunterricht kann unter anderem auf die Vielseitigkeit des Konzeptes zurückgeführt werden, die sich durch den normativen, den deskriptiven und den konstruktiven Aspekt der Grundvorstellungsidee ausdrückt.

Der normative Charakter von Grundvorstellungen als inhaltlich adäquate Deutungen ergibt sich aus stoffdidaktischen und stoffanalytischen Überlegungen (Greefrath et al. 2016a; vom Hofe 1995); ,normativ“" bezeichnet hier demnach nicht die willkürliche Setzung einer Norm, sondern eine auf mathematischen Inhalten basierende Formulierung.

In einer Vielzahl von Arbeiten werden Grundvorstellungen zu mathematischen Begriffen formuliert, die relevant für inhaltliche Kernbereiche des schulischen Mathematikunterrichts der Primar- und Sekundarstufe sind (Prediger 2010). Darunter sind Grundvorstellungen zu Grundrechenarten natürlicher Zahlen (Wartha und Schulz 2018), zum Proportionalitäts- und Prozentbegriff (Hafner 2012), zum Messen von Größen (Griesel 2016), zum Bruchzahlbegriff (Blum und Wiegand 1998; Malle 2004; Wartha 2007), zum Funktionsbegriff (Greefrath et al. 2016a; Malle 2000; Stölting 2008), zum Logarithmus (Weber 2013, 2016), zur Ableitung und zum Integral (Blum und Kirsch 1979; Greefrath et al. 2016a), zum Wahrscheinlichkeitsbegriff (Malle und Malle 2003), u. a.

In diesen Arbeiten wird deutlich, dass es zu einem mathematischen Begriff in den meisten Fällen nicht nur genau eine Grundvorstellung gibt; vielmehr existieren verschiedene inhaltlich verbundene Grundvorstellungen, die eine Art semantisches Netz bilden. Die Kenntnis der Grundvorstellungen und ihrer Verbindungen wird auch als Grundverständnis des jeweiligen Begriffs bezeichnet (Bender 1991; vom Hofe 2003a).

Weiterhin greift das Grundvorstellungskonzept auch auf einer deskriptiven Ebene: Auf der Basis von Schülerinnen- und Schülerdokumenten und -aussagen werden individuelle Lernendenvorstellungen rekonstruiert. Hierbei steht nicht die Frage im Zentrum, $o b$ eine Grundvorstellung ausgebildet wurde, sondern wie individuelle Vorstellungen detailliert beschrieben werden können (vom Hofe 1995). Die aus normativer Perspektive formulierten Grundvorstellungen werden hier als theoretischer Hintergrund und Orientierung für eine Einordnung und Bewertung der individuellen Vorstellungen herangezogen (vom Hofe 1995; vom Hofe und Blum 2016). ${ }^{2}$ So kann erhoben werden, inwieweit Schülerinnen und Schüler über tragfähige Vorstellungen verfügen und auf welche Weise individuelle Vorstellungen der Lernenden Erklärungen für systematische Fehler darstellen (z.B. Hafner 2012; Stölting 2008; Wartha 2007; Wessel 2015). In solchen Arbeiten wird insbesondere deutlich, inwieweit normativ formulierte Grundvorstellungen tatsächliche Relevanz für die Erklärung von Lern- und Denkprozessen haben (Weber 2013). Andersherum können die analysierten Lernendenvorstellungen Impulse für eine Um- oder Neuformulierung weiterer

\footnotetext{
2 Im Sinne einer deutlicheren Trennung dieser zwei Ebenen ist in der Physikdidaktik der Begriff „Schülervorstellung" verbreitet (z. B. Schecker und Duit 2018). Für eine kritische Betrachtung der deskriptiven Ebene sei z. B. auf Hanke und Schäfer (2017), Schacht (2012) und Vogel (2016) verwiesen.
} 
Grundvorstellungen sein (Wartha 2007). Für diese Formulierungen kann im Rahmen der Grundvorstellungsidee auf das Konzept der Subjektiven Erfahrungsbereiche nach Bauersfeld (1983) zurückgegriffen werden (vom Hofe 1995). Diese berücksichtigen ,individuelle und situationsspezifische Aspekte“ der Lernendenvorstellungen und gelten damit als „Mittel zur deskriptiven Analyse“ (vom Hofe 1995, S. 112), wobei in vom Hofes Interpretation Erfahrungsbereiche einer größeren Lernendengruppe hinsichtlich einer intersubjektiven Nachvollziehbarkeit der Lernendenerfahrungen erweitert werden.

Der Vergleich der deskriptiv ermittelten individuellen Vorstellungen und der normativ hergeleiteten Richtlinien ermöglicht die Gestaltung mathematischer Lerngelegenheiten, die die Differenzen zwischen Ist-Stand und wünschenswerten inhaltlichen Deutungen aufgreifen. In dieser gemeinsamen Betrachtung zeigt sich der konstruktive Aspekt des Grundvorstellungskonzeptes (vom Hofe 1995). Wie die Ausbildung tragfähiger Vorstellungen umgesetzt werden kann, wird in Fachzeitschriften mit Arbeitsmaterialien (z. B. vom Hofe 2003b; Frohn und Salle 2017) oder bei der Entwicklung digitaler Lernumgebungen (z. B. Etzold 2019; Reinhold 2019) in den Blick genommen.

Vorstellungen zu mathematischen Objekten und Sachverhalten nicht nur aus einer deskriptiven, sondern zunächst auch aus einer normativen Perspektive zu analysieren, grenzt das Grundvorstellungskonzept von vielen in der internationalen Diskussion verbreiteten Theorien $\mathrm{ab}$, insbesondere von der häufig rezipierten Theorie des concept image. Im Folgenden wird das Grundvorstellungskonzept diesbezüglich eingeordnet.

\subsection{Concept image, concept definition, conceptual change und deren Einordnung in das Grundvorstellungskonzept}

Das Grundvorstellungskonzept hat seinen Ursprung in der Rechendidaktik der Volksschule und den Bemühungen um Verständnisorientierung im Gymnasium (vom Hofe 1995; vom Hofe und Blum 2016) und prägt damit vor allem die deutschsprachige Stoffdidaktik. Die Forschung zu Lernendenvorstellungen hat länderübergreifend eine lange Tradition, die dazu führte, dass sich international verschiedene Konzepte zur Beschreibung von Vorstellungen entwickelt haben. Besondere Popularität hat hierbei die von Vinner und Hershkowitz (1980) sowie Tall und Vinner (1981) entwickelte Theorie von concept image und concept definition erlangt. ${ }^{3}$ Neben Definitionen - den concept definitions - für mathematische Lernprozesse, betrachten Tall und Vinner (1981) mit dem concept image sämtliche kognitiven Strukturen, die mit einem mathematischen Begriff verbunden werden, beispielsweise Darstellungen und Eigenschaften der Begriffe sowie insbesondere ausgebildete Vorstellungen (Klinger 2019).

Sowohl die Grundvorstellungstheorie als auch die Theorie zum concept image ermöglichen die Fokussierung auf Veränderungen von bereits ausgebildeten Vorstellungen (Klinger 2019). Neben der Identifizierung zentraler Vorstellungen zu

\footnotetext{
3 Für eine ausführliche Erläuterung zentraler Gemeinsamkeiten und Unterschiede beider Theorien sei auf Klinger (2019) und Greefrath et al. (2016b) verwiesen.
} 
mathematischen Begriffen aus normativer oder deskriptiver Perspektive wird der Übergang zwischen verschiedenen Stadien der Begriffsbildung thematisiert (z.B. Reinhold 2019). Mit diesen Übergängen verbundene Veränderungen von Vorstellungen werden bisher vor allem mit Hilfe der conceptual change-Theorie erklärt (z. B. Juter 2010; Vosniadou und Verschaffel 2004). In diesen Studien wird ein conceptual change nicht als Austausch, sondern als Reorganisation der vorhandenen Vorstellungen aufgefasst (Duit und Treagust 2003); je nach Einordnung der bereits bei den Lernenden vorhandenen Vorstellungen lässt sich demnach ein conceptual change zum einen mit dem Umbruch (Wartha 2007) und zum anderen mit dem Ausbilden von Grundvorstellungen (Kleine et al. 2005; vom Hofe und Blum 2016; Wartha 2007) in Beziehung setzen (Prediger 2008; Reinhold 2019). Im zweiten Fall wird conceptual change als der Übergang von vorunterrichtlichen Vorstellungen zu wissenschaftlich fundierten Vorstellungen - den Grundvorstellungen als normativen Leitlinien - verstanden.

Obwohl die normativ-inhaltliche Komponente das Grundvorstellungskonzept von vielen Konzepten zur Beschreibung von Vorstellungen abgrenzt, besteht oftmals die Frage, auf welche Weise konkrete normative Überlegungen zum Grundvorstellungskonzept vorgenommen werden. Zwar wird in einigen Arbeiten der Herleitungsprozess in Teilen beschrieben, explizit dargestellt oder kommentiert (z.B. Greefrath et al. 2016a; Weber 2013, 2016), ein theoretisch fundiertes Rahmenmodell mit einer allgemeinen Einordnung des Herleitungsprozesses in die Grundvorstellungstheorie liegt jedoch nicht vor. Um solch einen Verfahrensrahmen vorzuschlagen, betrachten wir im Folgenden zunächst Grundlagen bisheriger Herleitungen.

\section{Theoretische Grundlagen für die Herleitung von Grundvorstellungen}

\subsection{Didaktisch orientierte Sachanalysen und Didaktische Phänomenologie}

Um mathematische Inhalte für unterrichtliche Vorgehensweisen zu konkretisieren und mathematische Lernprozesse zu organisieren, setzen viele Arbeiten, insbesondere aus den 70er- und 80er-Jahren, didaktisch orientierte mathematische Sachanalysen ein (Griesel 1971; Vollrath 1987). Das Grundanliegen stoffdidaktischer Analysen im Sinne Kirsch (2000) besteht im Zugänglichmachen mathematischer Inhalte. Dies kann nach Kirsch (2000) vor allem durch eine Konzentration auf den mathematischen Kern, durch die Berücksichtigung der Begriffsgeschichte oder von Alltagsbezügen, durch das Anerkennen und Aktivieren von Vorwissen oder durch ein Wechseln der Repräsentationsform gelingen. Sachanalysen können über die Analyse einzelner Unterrichtsgegenstände hinaus auch auf die inhaltliche Strukturierung und Durchdringung größerer mathematischer Themenfelder gerichtet sein (z. B. Griesel 1970, 2016; Holland 1974; Kirsch 1987).

Während in den oben aufgeführten Beispielen oftmals konkrete unterrichtliche Ziele im Mittelpunkt stehen, hebt Vollrath (1987) die Sachanalyse auch explizit als mögliches Instrument zur Erforschung des mathematischen Lernens an sich heraus. Dies kann für ihn vor allem durch eine Einbettung in die didaktische Phänomenologie nach Freudenthal (1983) gelingen. Zentrale Phänomene werden dabei auf das 
Lehren und Lernen von Mathematik bezogen (Freudenthal 1983; Hußmann und Prediger 2016). Es wird dabei hinterfragt, wo und wie Begriffe, Strukturen und Ideen in mathematischen oder alltäglichen Zusammenhängen (Phänomenen) in Erscheinung treten und wo die Begriffe ursprünglich angewendet wurden.

Zentral im Hinblick auf Grundvorstellungen ist bei Freudenthal (1983) zum einen das Manipulieren mentaler Objekte, die er als intuitions nach Fischbein (1987) auffasst, und die einer tragfähigen Begriffsbildung vorausgehen. Zum anderen werden diese mentalen Objekte durch die Anwendung mathematischer Konzepte geprägt. Ausgewählte mentale Objekte im Sinne Freudenthals (1983) können demnach auch Anknüpfungspunkte für eine Ausbildung von Grundvorstellungen sein.

Der Sachanalyse kommt nach Vollrath (1987, S. 251) im Rahmen einer didaktischen Phänomenologie zusammenfassend die Aufgabe zu, auszubildende mentale Objekte und Vorstellungen zu mathematischen Sachverhalten herzuleiten, ihre „mathematische Tragfähigkeit“ zu überprüfen und diese in ein Netz von Zusammenhängen zu stellen; sie stellt so eine generelle Leitlinie für die Herleitung von Grundvorstellungen bereit (Weigand 2015).

Ein Analyseverfahren, das in ähnlicher Weise versucht, tragfähige mentale Repräsentationen zu identifizieren, ist die conceptual analysis (Thompson 2008). Auch hier wird nach der Festlegung von Begriffen das Ineinandergreifen von empirischen und sachanalytischen Erkenntnissen bei der präskriptiven Formulierung von zentralen Ideen betont. Ein ähnliches Zusammenwirken von Empirie und Theorie findet sich im Bereich der fachdidaktischen Entwicklungsforschung, wobei hier die Konzeption fundierter Lehr-Lern-Arrangements, die empirische Erforschung von Lernprozessen und die Theorieentwicklung noch stärker im Rahmen eines Kreislaufmodells verzahnt sind (Prediger et al. 2015).

\subsection{Herleitung von Grundvorstellungen in der Literatur}

Arbeiten, in denen eine ausführliche Herleitung von Grundvorstellungen als normative Leitlinien dargestellt wird, nehmen im Sinne der obigen Ausführungen eine Analyse mathematischer Inhalte und korrespondierender Phänomene vor (z. B. Greefrath et al. 2016a; Roos 2020; Weber 2013, 2016). Dabei stehen insbesondere die Betrachtung zentraler Begriffsanwendungen sowohl in Sach- als auch in mathematischen Kontexten, Verbindungen zu anderen mathematischen Konzepten und die Betrachtung der Begriffsgeschichte im Fokus (vom Hofe und Blum 2016).

Ein Bereich, in dem bereits relativ früh konkrete Grundvorstellungen formuliert wurden, ist die elementare Bruchrechnung. Den Kern bilden hier drei Grundvorstellungen zum Bruchzahlbegriff (Blum und Wiegand 1998; Wartha 2007). Basierend auf einem historischen Abriss zur didaktischen Erforschung der Bruchrechnung sowie einer strukturierten Betrachtung der Verwendungssituationen von Brüchen leitet Wartha (2007) die Anteilsvorstellung, die Verhältnisvorstellung und die Operatorvorstellung her. Mit der Analyse von Schülerlösungen und insbesondere dem Herausarbeiten von typischen fehlerhaften Vorstellungen werden hier zudem empirische Studien einbezogen. 
Bei Weber (2013) wird ein sachanalytisches Vorgehen zur Herleitung von Grundvorstellungen zum Logarithmus dargestellt. ${ }^{4}$ Über die Berücksichtigung der Begriffshistorie und alltäglichen sowie innermathematischen Kontexten und Anwendungen werden Grundvorstellungen abgeleitet. Dieses Vorgehen ,ist von vornherein auf ein didaktisches Ziel gerichtet, sollte sich an der Anwendungsdimension des mathematischen Inhalts und am Erfahrungshorizont des Schülers orientieren und stellt somit eine grundsätzliche didaktische Entscheidung dar" (vom Hofe 1995, S. 123f.). Hier wird deutlich, dass es sich bei der Herleitung von Grundvorstellungen um einen konstruktiven Prozess handelt, bei dem die resultierenden Grundvorstellungen durch Entscheidungen der Forschenden geprägt sind. Im Anschluss an die Formulierung der Grundvorstellungen schildert Weber (2013, S. 94) mittels analysierter Schüleräußerungen „Indizien für die Tragfähigkeit der genannten Grundvorstellungen“.

Bei der Analyse der mathematischen Inhalte kann auch über sogenannte Aspekte eines mathematischen Begriffs vorgegangen werden (Greefrath et al. 2016a; Roos 2020). Als Aspekt eines mathematischen Begriffs sehen Greefrath et al. (2016a, S. 17) ,einen Teilbereich des Begriffs, mit dem dieser fachlich charakterisiert werden kann“.5 Die Aspekte werden auf Basis einer Analyse verschiedener Begriffsdefinitionen abgeleitet. Über Anwendungen von Begriffen in der Mathematik oder in Situationen mit Umweltbezug sowie einer Analyse der historischen Begriffsentwicklung ordnen Greefrath et al. (2016a, 2016b) den Aspekten verschiedene Grundvorstellungen zu.

Hußmann und Prediger (2016, S. 34) schlagen einen umfassenden Ansatz zur „Spezifizierung und Strukturierung mathematischer Lerninhalte“ vor: Im Rahmen des specifying - der generellen Identifikation inhaltlicher Aspekte - werden Grundvorstellungen als wichtiger Bestandteil der semantischen Ebene, die die Bedeutung der zu lernenden Konzepte aufgreift, aufgeführt. Zwar werden bei Hußmann und Prediger (2016) keine neuen Grundvorstellungen hergeleitet; in der konkreten Identifikation von Grundvorstellungen zu exponentiellem Wachstum zeigen Autor und Autorin jedoch auf, wie die Beziehungen zwischen Anwendungen, Grundvorstellungen und den formalen mathematischen Grundlagen zentraler Bestandteil einer Auswahl und Anordnung von Grundvorstellungen als Leitlinien für den Unterricht sein können. Zudem wird auch die Bedeutung empirischer Studien im Rahmen des specifying herausgearbeitet: Das Rekonstruieren der Perspektiven von Lernenden ermöglicht fundamentale Beiträge zu epistemologisch ausgerichteten Analysen von Lernprozessen.

Auch bei Wessel (2015) werden keine neuen Grundvorstellungen zur Subtraktion formuliert, jedoch Hinweise zur Herleitung von Grundvorstellungen gegeben. Bevor die in der Literatur bereits beschriebenen Grundvorstellungen dargestellt werden, wird auf den Aufbau und die Struktur von Subtraktionsaufgaben (syntaktische Struktur) sowie auf die den Aufgaben zugrundeliegenden Sachsituationen (seman-

\footnotetext{
${ }^{4}$ Die beschriebene Herleitung wird in Weber (2016) um eine operationale bzw. strukturelle Perspektive erweitert.

5 Die verwendete Bezeichnung „Aspekt“ wird in der Literatur unterschiedlich ausgelegt. Greefrath et al. (2016b) nehmen auf diese verschiedenen Auslegungen Bezug und erläutern dies zudem in Greefrath et al. (2016a, S. 254) u. a. exemplarisch an der Integralrechnung.
} 
tische Struktur) eingegangen. Im Anschluss an die Analysen wird angemerkt, dass „die Identifikation beziehungsweise Klassifikation der syntaktischen und semantischen Strukturen der Operationen dazu dienen kann Grundvorstellungen zu diesen zu bestimmen“ (Wessel 2015, S. 37 f.).

\subsection{Zusammenfassung}

Allen genannten Arbeiten ist ein sachanalytisches Vorgehen gemeinsam, in dem die Phänomenologie der jeweiligen Begriffe und die mathematischen Definitionen den Kern des Verfahrens darstellen. Die Schwerpunktsetzung, mit der innermathematische Anwendungen, Anwendungen mit Umweltbezug sowie die Begriffsentwicklung einbezogen werden, variiert in den aufgeführten Beiträgen. Als grundlegend didaktisch zielgerichtetes Vorgehen wird bei der Analyse der „Erfahrungshorizont“ (vom Hofe 1995, S. 123) bzw. die „Erfahrungswelt“ (Weber 2013, S. 88) der Lernenden berücksichtigt.

In den dargelegten Arbeiten wird neben den ausgeführten normativen Analysen zudem deutlich, welche Rolle empirische Ergebnisse bei der Formulierung von Grundvorstellungen einnehmen können. Zum einen können deskriptive Untersuchungen gemeinsam mit einem sachanalytischen Vorgehen Grundlage für Neu- und Umformulierungen sein. Zum anderen stellen empirische Ergebnisse zentrale Elemente einer nachgelagerten Bewährung der konkretisierten Grundvorstellungen dar. Dabei können sowohl Hinweise, dass die hergeleiteten Grundvorstellungen Gemeinsamkeiten mit den Vorstellungen der Lernenden aufweisen, als auch die Identifikation von Fehlstrategien, die mit den neu formulierten Leitlinien Zusammenhänge aufweisen, wichtige Relevanzkriterien sein (Wartha 2007; Weber 2013).

\section{Ein Vorgehen zur Herleitung von Grundvorstellungen als normative Leitlinien}

Die Betrachtungen aus Abschn. 3 zeigen, dass Sachanalysen und insbesondere der Formulierung von Grundvorstellungen eine Eingrenzung der Inhalte vorausgeht (Greefrath et al. 2016a; Hußmann und Prediger 2016; Vollrath 1987; Weber 2013). Somit sind die zentralen mathematischen Konzepte, deren Gültigkeitsbereich und der Bezugsrahmen der abzuleitenden Grundvorstellungen in einem ersten Schritt zu bestimmen.

Das folgende sachanalytische Vorgehen, eingebettet in die Phänomenologie Freudenthals (1983), ist geprägt von einem steten Aufeinanderbeziehen von Mathematik und Phänomenen (s. Abschn. 3.1). Die zentralen Bestandteile der mathematischen Definitionen, die im Rahmen dieses Prozesses herausgeschält werden, fassen wir im Folgenden als Kernelemente des jeweils betrachteten mathematischen Begriffs auf. Diese Kernelemente ergeben sich aus einer Zuordnung von zentralen mathematischen Bestandteilen einer Definition (Objekte, Operationen, Eigenschaften ...) und zentralen Bestandteilen von Phänomenen (Begriffsanwendungen, -entwicklung ...). Kernelemente sind jene zentralen Elemente des mathematischen Begriffs, die sich in den korrespondierenden Phänomenen wiederfinden. Auf dieser Basis werden ide- 
altypische mentale Repräsentationen - also Grundvorstellungen - als vermittelnde Elemente zwischen Mathematik und den Anwendungen, identifiziert. Die durch den Zuordnungsprozess ermöglichte Identifikation von Kernelementen im Abgleich mit empirischen Ergebnissen sehen wir demnach als zweiten Schritt, welcher das Herzstück des vorgeschlagenen Verfahrens darstellt.

Den theoretischen Ausführungen folgend (s. Abschn. 2) bilden solche Zuordnungen die Basis für die Formulierung von Grundvorstellungen; diese Formulierung stellt demnach den folgenden dritten Schritt dar. An dieser Stelle sind zudem die Beziehungen zwischen den Grundvorstellungen zu klären, um letztere beispielsweise hinsichtlich möglicher Grundvorstellungsumbrüche und ihr Zusammenwirken bzgl. des Grundverständnisses des Begriffs zu untersuchen (Bender 1991; Wartha 2007; s. Abschn. 2.1).

Sind die Ergebnisse dieser Schritte aus Sicht der Forschenden (vorerst) zufriedenstellend, kann der Bezugsrahmen der hergeleiteten Grundvorstellungen wieder aufgegriffen und weiter präzisiert werden; dies stellt den vierten Schritt dar. Wie in Abschn. 3.2 und 3.3 beschrieben schließt eine Bewertung der didaktischen Relevanz den Herleitungsprozess ab; diese ist demnach im fünften Schritt vorzunehmen.

Die beschriebenen Schritte sind als flexibel zu durchlaufender Kreislauf zu verstehen. So kann die nähere Untersuchung hergeleiteter Grundvorstellungen (Schritt 3) zu einer erneuten sachanalytischen Analyse (Schritt 2) führen oder eine Veränderung der Festlegungen bedingen (Schritt 1). Zudem könnten empirische Studien zu individuellen Vorstellungen der Lernenden oder zur Eignung grundvorstellungsbasierter Unterrichtskonzeptionen, die im Rahmen der Relevanzklärung durchgeführt werden (Schritt 5), eine neue Durchführung des beschriebenen Verfahrens unter veränderten Grundbedingungen veranlassen.

Zusammengefasst besteht das vorgeschlagene Vorgehen aus fünf Schritten:

1. Bestimmung von Richtlinien für den Herleitungsprozess

2. Sachanalyse des mathematischen Begriffs und seiner Phänomene sowie Einbezug empirischer Ergebnisse

3. Formulierung konkreter Grundvorstellungen und Analyse des Grundverständnisses

4. Präzisierung des Bezugsrahmens

5. Feststellung und Bewertung der didaktischen Relevanz

\subsection{Erster Schritt: Bestimmung von Richtlinien für den Herleitungsprozess}

$\mathrm{Zu}$ Beginn des Herleitungsprozesses werden einige Richtlinien festgelegt, die die folgenden Schritte fokussieren.

Bestimmung der zentralen mathematischen Konzepte und ihrer Gültigkeitsbereiche Zunächst werden die mathematischen Begriffe festgelegt, zu denen Grundvorstellungen formuliert werden sollen. Zusätzlich wird bestimmt, welcher Gültigkeitsbereich der Begriffe im Folgenden betrachtet werden soll (s. Abschn. 2.1). Dies kann insbesondere zu einer Einschränkung der inhaltlichen Reichweite für weitere Analysen führen. 
Erste Bestimmung des Bezugsrahmens Damit Lernende Grundvorstellungen zu mathematischen Begriffen aufbauen können, sind Kenntnisse, Kompetenzen und Vorstellungen zu anderen Begriffen notwendig. Somit wird in diesem Schritt der Bezugsrahmen der zu formulierenden Grundvorstellung abgesteckt (s. Abschn. 2.1). Auf dieser Basis kann beispielsweise in Schritt 2 entschieden werden, welche Definitionen und Phänomene in die weiteren Analysen einbezogen und welche verworfen werden können. ${ }^{6}$

Da das Grundvorstellungskonzept im Ursprung ein auf den schulischen Mathematikunterricht ausgerichtetes Konzept ist (vom Hofe 1995), können die den Lernenden bekannte Inhalte auch durch curriculare Vorgaben mitbestimmt werden. Generell ist die Bestimmung des Bezugsrahmens jedoch auch ausschließlich auf Basis fachlichinhaltlicher Überlegungen möglich, wie beispielsweise entlang fundamentaler Ideen oder der Begriffsgeschichte sowie unter Einbezug einschlägiger empirischer Studien (Greefrath et al. 2016a; Vohns 2005).

Der in diesem Schritt abgesteckte Bezugsrahmen kann in den folgenden Schritten erweitert oder eingeschränkt werden (s. Schritt 4).

\subsection{Zweiter Schritt: Sachanalyse des mathematischen Begriffs und seiner Phänomene sowie Einbezug empirischer Ergebnisse}

In diesem Schritt werden zunächst die für die Herleitung von Grundvorstellungen notwendigen mathematischen Grundlagen (Abschn. 4.2.1) und die dazugehörigen Phänomene (Abschn. 4.2.2) mittels einer Sachanalyse in den Blick genommen (s. Abschn. 3.1). Diese Analysen werden als zwei ineinandergreifende Komponenten immer wieder aufeinander bezogen (Abschn. 4.2.3). Den Rahmen dieser Analysen legen die Richtlinien aus Schritt 1 fest, auf deren Basis Definitionen und Phänomene ausgewählt, spezifiziert oder verworfen werden. Weiterhin werden diese inhaltlich gewonnenen Ergebnisse zu Ergebnissen empirischer Untersuchungen in Beziehung gesetzt, wobei letztere sowohl Ergänzung als auch Ausgangspunkt für diesen zweiten Schritt sein können.

\subsubsection{Analyse der mathematischen Konzepte}

Identifikation, Analyse und Auswahl von geeigneten Definitionen Es werden verschiedene Definitionen der zentralen Begriffe zusammengetragen. Anschließend wird analysiert, welche mathematischen Objekte, Verfahren etc. für das Verständnis der identifizierten Definitionen benötigt werden. Die Auswahl geeigneter Definitionen wird nicht vor dem Hintergrund einer konkreten Unterrichtsstrukturierung vorgenommen; vielmehr werden alle vor dem Hintergrund des Bezugsrahmens potenziell relevanten Definitionen beibehalten. Außerdem werden die zentralen mathematischen Bestandteile einer Definition (Objekte, Operationen ...) herausgearbeitet,

\footnotetext{
6 s. z. B. bei Padberg und Wartha (2017) zum Ausschluss des Äquivalenzklassenkonzeptes bei der Analyse von Grundvorstellungen rationaler Zahlen.
} 
die im Folgenden durch Einbettung in die Phänomene als Kernelemente charakterisiert werden (s. Abschn. 4.2.4). ${ }^{7}$

Herausarbeiten des Zusammenhangs von Definitionen Die analysierten Definitionen werden auf Zusammenhänge und Unterschiede hin analysiert. Es empfiehlt sich, leicht unterschiedliche Definitionen zunächst getrennt zu betrachten, da sich auch kleine Unterschiede durch die Einbettung in die Phänomene als wichtige Distinktionsmerkmale herausstellen und so unterschiedliche Grundvorstellungen entstehen können.

\subsubsection{Sammlung und Analyse relevanter Phänomene}

Identifikation und Analyse von Anwendungen Kernfragen dieser Analyse beziehen sich auf typische Situationen, in denen die mathematischen Inhalte in Erscheinung treten (s. Abschn. 3.1). So muss geklärt werden, wo in der Umwelt, in verwandten Disziplinen oder in innermathematischen Zusammenhängen (z. B. bei bestimmten mathematischen Operationen, beim Beweisen und Problemlösen) die ausgewählten Konzepte wirksam werden. Auch Aufgaben oder Lösungsbeispiele in Lehrwerken liefern Hinweise (s. Abschn. 3.2). Abschließend muss die Rolle der mathematischen Konzepte in den identifizierten Phänomenen im Hinblick auf die spätere Klassenbildung und die Herausarbeitung der Kernelemente analysiert werden.

Analyse der Begriffsgenese und historischen Entwicklung Zur Analyse der Phänomene gehören ebenfalls die Fragen, auf welchen Konzepten die ausgewählten Konzepte in ihrer Entwicklung aufbauten, welche Anwendungsfelder und Disziplinen Motivation zur Entwicklung der Konzepte waren und welche Etappen der Begriffsentwicklung sich identifizieren lassen (s. Abschn. 3.1).

Analyse typischer Darstellungen Darstellungen spielen für die Ausbildung von Grundvorstellungen und damit für die hier betrachtete Analyse eine zentrale Rolle (Bruner 1964; Stölting 2008). Insbesondere bei Übersetzungen von einer Darstellung in eine andere werden Grundvorstellungen benötigt bzw. ausgebildet. Demnach ist zentral,

- welche Darstellungen eines Begriffs auf welche Weise beim mathematischen Arbeiten und in zentralen Phänomenen auftreten,

- welche Übersetzungsprozesse diesbezüglich wichtig sind,

- welche Darstellungen in der Begriffsentwicklung fundamental waren (Padberg und Wartha 2017; Weber 2013).

\footnotetext{
7 An dieser Stelle ist zudem eine Analyse von Aspekten mathematischer Konzepte im Sinne einer fachlichen Charakterisierung möglich, falls solche vorliegen oder formuliert werden sollen (Greefrath et al. 2016b).
} 


\subsubsection{Einbezug empirischer Ergebnisse}

Da der Einbezug der Lernendenperspektive grundlegend für die Formulierung von Grundvorstellungen ist, müssen Ergebnisse entsprechender Studien berücksichtigt werden (Hußmann und Prediger 2016; Thompson 2008; Weber 2013). Empirische Ergebnisse können Hinweise darauf geben, welche Bedeutung Phänomene für Schülerinnen und Schüler haben. Weiterhin sind auch Forschungsarbeiten zu berücksichtigen, die mentale Repräsentationen z. B. im Rahmen der Theorie des concept image oder des conceptual change fokussieren.

Um zu bestimmen, welche Implikationen empirische Studien für den Herleitungsprozess von Grundvorstellungen haben, ist zu berücksichtigen, über welches Vorwissen die Probandinnen und Probanden in diesen Studien verfügen, wie weit der Gültigkeitsbereich der zentralen Konzepte gefasst ist und wie eventuelle Interventionen konzipiert wurden.

\subsubsection{Zusammenschau}

Zuordnung von Definitionen und Phänomenen sowie Identifikation von Kernelementen Die ausgewählten und analysierten Definitionen und Phänomene werden immer wieder geordnet und miteinander in Zusammenhang gebracht. Durch einen Vergleich der Bestandteile der mathematischen Definitionen und ihrer jeweiligen Einbettung in die zugeordneten Phänomene können sogenannte Kernelemente der mathematischen Konzepte gefunden und formuliert werden, die ausschlaggebend für die folgende Klassenbildung sind.

Klassenbildung Die Formulierung der neuen Grundvorstellungen basiert auf der Bildung von Klassen, in denen sowohl die mathematischen Objekte als auch die Phänomene unter Berücksichtigung empirischer Ergebnisse gemeinsam betrachtet werden.

Als verbindende Elemente zwischen den Definitionen und den Phänomenen werden die identifizierten Kernelemente zur Charakterisierung der Klassen genutzt. Eine Klasse wird gebildet, indem die im vorherigen Teilschritt getroffenen Zuordnungen und damit die identifizierten Kernelemente gruppiert werden. Ein Kernelement des mathematischen Konzeptes kann in unterschiedlichen Phänomenen und damit auch in verschiedenen Klassen wirksam werden.

Die Auswahl, welche Kernelemente ausschlaggebend für das Bilden einer Klasse sind, ist eine Entscheidung, die auf Basis der zu Beginn formulierten Richtlinien, der Ergebnisse der obigen Analysen und empirischen Ergebnissen von den Forschenden getroffen werden muss. Empirische Ergebnisse können zudem auch Ausgangspunkt für die Bildung der Klassen sein, wenn beispielsweise bereits vor der sachanalytischen Präzisierung Vorschläge für empirisch gewonnene Vorstellungen vorliegen.

Die besagten Klassen bilden sich weder kanonisch noch eindeutig; es sind verschiedene Klassenbildungen denkbar, wenngleich das konsequente Arbeiten mit den beschriebenen Richtlinien grundsätzlich eine deutliche Einschränkung von Freiheitsgraden bei der Formulierung bedeutet. 
Auswahl relevanter Klassen Um relevante Klassen zu identifizieren, sind mehrere Kriterien entscheidend. Es muss eingeschätzt werden,

- welche Ergebnisse für empirisch gewonnene individuelle Vorstellungen bereits vorliegen,

- ob sich die Klasse auf hinreichend reichhaltige bzw. relevante Phänomene bezieht,

- inwieweit bisher gebildete Klassen zusammengefasst werden können oder in mehrere Klassen zerfallen.

Das Entstehen und Verwerfen von Klassen sind Entscheidungen, die auf Grundlage der schon vorliegenden Klassen und vor dem Hintergrund der bisherigen Festlegungen aus Schritt 1 getroffen werden müssen.

\subsection{Dritter Schritt: Formulierung konkreter Grundvorstellungen und Analyse des Grundverständnisses}

Die formulierten Klassen sind Grundlage für die Formulierung der Grundvorstellungen.

Formulierung der Grundvorstellungen Um eine (neue) Grundvorstellung formulieren zu können, werden die Kernelemente für jede in Schritt 2 herausgearbeitete Klasse als Gesamtes betrachtet und die dahinterliegende idealtypische Repräsentation des betrachteten Begriffs festgehalten. Die Namen der Grundvorstellungen können u. a. auf Basis der Kernelemente gewählt werden. ${ }^{8}$

Durch eine Berücksichtigung des hier vorgeschlagenen Verfahrens genügen die (neu) formulierten Vorstellungen auf normativer Ebene den drei zentralen Eigenschaften des Grundvorstellungskonzeptes (s. Abschn. 2.1): Indem in den ersten beiden Schritten der Erfahrungshorizont der Lernenden durch den Bezugsrahmen grundlegend beachtet wurde, können die neu formulierten Leitlinien den Lernenden eine Anknüpfung an ihnen bekannte Handlungszusammenhänge und somit eine Sinnkonstituierung des Begriffs ermöglichen (erste Eigenschaft). Durch die Anknüpfung der Definitionen an analysierte Phänomene wird bedacht, dass die neuen Leitlinien zum mentalen Operieren auf Vorstellungsebene (zweite Eigenschaft) und zur Anwendung der mathematischen Objekte (dritte Eigenschaft) befähigen sollen.

Analyse des auszubildenden Grundverständnisses und Identifikation von Grundvorstellungsumbrüchen Um ein Grundverständnis eines Begriffs auszubilden, sollten nach vom Hofe (2003a) neben den zugehörigen Grundvorstellungen auch deren Verbindungen untereinander in den Blick genommen werden (s. Abschn. 2.2).

Je nachdem, wie Grundvorstellungen bei einer Vermittlung angeordnet werden (ob beispielsweise eine Grundvorstellung aufbauend auf einer anderen thematisiert

\footnotetext{
8 Wurden bei der mathematischen Analyse „Aspekte“ mathematischer Begriffe mit einbezogen, können diese bei einer entsprechenden Passung ebenfalls für die Benennung einer Grundvorstellung in Frage kommen (beispielsweise Zuordnungsaspekt und Zuordnungsvorstellung bei Folgen, Greefrath et al. 2016a; Vollrath 1989).
} 
wird), können Grundvorstellungsumbrüche auftreten (s. Abschn. 2.1). Die Beziehungen der neu formulierten Grundvorstellungen sind zentral für eine spätere unterrichtliche Nutzung.

\subsection{Vierter Schritt: Präzisierung des Bezugsrahmens}

Im Anschluss an die Formulierung der neuen Grundvorstellungen kann ihr bereits vorläufig bestimmter Bezugsrahmen präziser abgesteckt werden.

\section{Bestimmung von Grundkenntnissen zu den formulierten Grundvorstellungen}

Es werden Grundkenntnisse analysiert, die für eine Verwendung dieser Grundvorstellungen und damit insbesondere für das mentale Operieren und zur Anwendung des mathematischen Begriffs benötigt werden (s. Abschn. 2.1). Über den bereits vorläufig bestimmten Bezugsrahmen (s. Schritt 1) kann präzisiert werden, welche Grundkenntnisse vorhanden sein könnten bzw. sollten.

Bestimmung der Zusammenhänge zu anderen Grundvorstellungen aus dem Bezugsrahmen Basierend auf dem Bezugsrahmen (s. Schritt 1) werden Grundvorstellungen zu weiteren zentralen Begriffen identifiziert, die Voraussetzungen für die Tragfähigkeit und Anwendung der formulierten Grundvorstellungen darstellen.

\subsection{Fünfter Schritt: Feststellung und Bewertung der didaktischen Relevanz}

Die tatsächliche Relevanz der formulierten Grundvorstellungen sollte auf mehreren Ebenen bewertet werden (s. Abschn. 3.2 und 3.3).

Identifikation der Vorstellungen bei Lernenden sowie Identifikation von Fehlvorstellungen Inwieweit sich die normativ formulierten Leitlinien auch bei Lernenden als individuelle Vorstellungen identifizieren lassen und inwieweit sie Erklärungsmodelle für deren Handeln darstellen, sind wichtige zu klärende Fragen für die Relevanz der formulierten Grundvorstellungen (s. Abschn. 3.2 und 3.3).

Zum einen sollten in empirischen Studien individuelle Vorstellungen rekonstruiert werden und anschließend evaluiert werden, inwieweit diese Vorstellungen Gemeinsamkeiten mit formulierten Grundvorstellungen aufweisen. Auch kann sich herausstellen, dass die formulierten Leitlinien einer weiteren Ausdifferenzierung bedürfen, da sich beispielsweise bisher unbeachtete Phänomene im Erfahrungshorizont der Lernenden als grundlegend für ihre Bedeutungsanknüpfung erweisen. Zum anderen können durch Analysen von Fehlern bzw. Fehlermustern, basierend auf den hergeleiteten Grundvorstellungen, Fehlvorstellungen formuliert und identifiziert werden, die im Sinne vom Hofes (1995) inadäquate inhaltliche Deutungen der Begriffe darstellen. Obgleich empirische Ergebnisse im beschriebenen Herleitungsprozess berücksichtigt werden, sind die hier beschriebenen Untersuchungen zentral für die Evaluation der normativen Leitlinien und deren notwendige Anknüpfung an die Denkprozesse der Lernenden (z. B. Hafner 2012; Stölting 2008; Wartha 2007). 
Bewährung in der Unterrichtsgestaltung Über deskriptive Studien hinaus ist der Nutzen der neu formulierten Leitlinien in Lehr-Lern-Situationen eine weitere wichtige Bewährungsebene. Kernfragen sind hier, inwieweit die Grundvorstellungen Leitlinien für Unterrichtsgestaltung sein können bzw. in bestehende Unterrichtskonzepte eingebettet werden können (s. auch Hußmann und Prediger 2016) und inwieweit ein konstruktiver Umgang mit individuellen Vorstellungen realisiert werden kann (s. Abschn. 2.2).

\section{Zwei Beispiele für die Herleitung von Grundvorstellungen}

In den folgenden Abschnitten wird das Herleitungsverfahren exemplarisch an zwei Begriffen vorgestellt. Zuerst wird für den Sinusbegriff gezeigt, wie neue Grundvorstellungen zu einem Begriff hergeleitet werden, der im Rahmen von Grundvorstellungen bisher nicht betrachtet wurde. Anschließend wird für den Bruchzahlbegriff dargestellt, wie mit dem Herleitungsverfahren etablierte Grundvorstellungen rekonstruiert werden können. Hier wird ebenfalls deutlich gemacht, wie Formulierungen der Grundvorstellungen durch Entscheidungen der Forschenden beeinflusst und so gegebenenfalls ausdifferenziertere Grundvorstellungen formuliert werden könnten.

\subsection{Grundvorstellungen zum Sinusbegriff}

\subsubsection{Festlegungen, Sachanalyse des mathematischen Begriffs und seiner Phänomene sowie Einbezug empirischer Ergebnisse}

Für den ausgewählten Sinusbegriff wird zunächst ein Gültigkeitsbereich festgelegt, indem nur reelle Zahlen bzw. Winkel als Argumente zugelassen werden; diese Entscheidung und der weitere vorläufige Bezugsrahmen werden durch typische Inhalte der Sekundarstufe I bestimmt. Relevant sind hier unter anderem die Bruchrechnung, die Dreieckslehre, funktionale Zusammenhänge und elementare Gleichungsumformungen.

Die im Rahmen der obigen Festlegungen relevanten Definitionen sind

- Sinus eines Winkels $\alpha$ im rechtwinkligen Dreieck als Seitenverhältnis von Gegenkathete und Hypotenuse. Grundlegende Bestandteile dieser Definition sind ein rechtwinkliges Dreieck, der Winkel $\alpha$, die Seitenlängen von Gegenkathete und Hypotenuse, sowie das Verhältnis dieser Seitenlängen, ausgedrückt durch einen Quotienten bzw. eine reelle Zahl.

- Sinus eines Winkels $\alpha$ als $y$-Koordinate eines Punktes auf dem Einheitskreis. Grundlegende Bestandteile dieser Definition sind der Winkel $\alpha$, der Strahl, der durch $\alpha$ zusammen mit der $x$-Achse den Punkt $Q$ auf dem Einheitskreis festlegt, und die $y$-Koordinate von $Q$. Auf dieser Grundlage wird auf Basis des BogenmaBes die trigonometrische Funktion $\sin : \mathbb{R} \rightarrow[-1 ; 1]$ definiert.

Weitere Definitionen werden in den folgenden Analysen nicht berücksichtigt, da sie deutlich über den festgelegten Bezugsrahmen hinausgehen. In der ersten Definition werden für $\alpha$ nur Werte zwischen 0 und $90^{\circ}$ betrachtet. In diesem Zusammen- 
hang sind die einbeschriebenen Dreiecke Sonderfälle, da ausschließlich Dreiecke mit einer Hypotenusenlänge von eins betrachtet werden. Ein zentraler Unterschied der Definitionen ist, dass Sinus von $\alpha$ in der ersten Definition als Verhältnis von Seitenlängen entsteht, während Sinus im zweiten Fall der Wert einer Koordinate ist.

Aufgrund des Umfangs der Analysen beschränken wir uns im Weiteren auf solche relevanten Phänomene, die sich auf die Definition des Sinus eines Winkels an rechtwinkligen Dreiecken beziehen. Dies sind beispielsweise Triangulation bei der Vermessung der Erde oder bei Computergrafiken, Bestimmung von Schattenwürfen sowie Bestimmung von Winkeln und Strecken in Dreiecken, von Flussbreiten oder Baumhöhen (Filler 2006, 2018; Frohn 2020; Funk 2013; Malle 2001).

Die historische Begriffsentwicklung des Sinuskonzeptes hat ihren Ursprung in der Landvermessung und Architektur der Ägypter und Babylonier. Weitere wichtige Etappen dieser Entwicklung sind: Berechnungen von Schattenlängen und deren Verhältnisse in verschiedenen Kulturen in der Antike; Einbettung von Dreiecken in Kreise mit festen Radien zur Bestimmung der Sehnenlängen bei den alten Griechen; Erstellung trigonometrischer Tafeln mit verschiedenen Methoden; (Weiter-)Entwicklung der Konzepte formalerer trigonometrischer Funktionen in astronomischen Anwendungen, Navigation und Kalendarien, vom 17.-19. Jh. (z. B. Merzbach und Boyer 2011; Rogers und Pope 2016; Wußing 2008).

Typische Darstellungen sind die folgenden:

- Symbolisch: Das Verhältnis zweier entsprechender Seiten $a$ und $c$ wird durch $\sin (\alpha)=\frac{a}{c}$ berechnet. In der Gleichung $c \cdot \sin (\alpha)=a$ ist der Sinus eines Winkels der Faktor, der als Operator auf die Länge der Hypotenuse wirkt.

- Graphisch: Dreiecke in zwei- oder dreidimensionalen Koordinatensystemen, Dreiecke im Einheitskreis, Darstellungen von Funktionsgraphen.

- Tabellarisch: Tabellen konkreter Sinuswerte zu Winkeln bzw. Kreisbögen, Tabellen als Darstellungen trigonometrischer Funktionen.

Empirische Ergebnisse geben verschiedene Hinweise für die Bedeutung von Seitenverhältnissen beim Verständnis des Sinus eines Winkels im rechtwinkligen Dreieck. In Fallstudien konnte gezeigt werden, dass Lernende mithilfe solcher Seitenverhältnisse argumentieren (z. B. Brown 2005). Schwierigkeiten zeigen sich beispielsweise bei dem Umgang mit Verhältnissen und der Bruchrechnung (Blackett und Tall 1991; Kendal und Stacey 1996) sowie bei der Zuordnung der Gleichungsbestandteile und dem Bestimmen von Lösungen in trigonometrischen Beziehungen (Kendal und Stacey 1996; Müller 1997).

Durch eine Zuordnung von Definitionen und Phänomenen sowie durch eine Einordnung der Analysen in die Empirie lassen sich mehrere Kernelemente des Sinuskonzeptes in Bezug auf rechtwinklige Dreiecke identifizieren. Auf diesen Elementen aufbauend können zwei Klassen identifiziert werden:

\section{Erste Klasse: Seitenverhältnis in rechtwinkligen Dreiecken (ohne Projektion)}

Der Sinus eines Winkels dient hier der Berechnung von Seitenverhältnissen in bestehenden rechtwinkligen Dreiecken (z. B. bei der Vermessung von Höhen und anderen Maßen, bei der Bestimmung fehlender Größen in Dreiecken, etc.).

Entscheidende Kernelemente sind: 
- das rechtwinklige Dreieck als gegebene Dreiecksfigur, die nicht aus einer Projektion hervorgeht,

- die Hypotenuse, die eine Seitenlänge darstellt,

- die Gegenkathete, die eine weitere Seitenlänge darstellt,

- der Ausdruck $\sin (\alpha)$ als Verhältnis der Seitenlängen von Gegenkathete und Hypotenuse.

Zweite Klasse: Projektionsfiguren bei orthogonaler Projektion Der Sinus eines Winkels dient hier zur Berechnung von Projektionsfaktoren bzw. projizierten Längen durch spezifische orthogonale Projektionen von Objekten und Seiten in einer durch die Projektion entstehenden rechtwinkligen Dreiecksfigur (z. B. bei der Berechnung von Schattenlängen und Lichtintensitäten, bei der Koordinatenbestimmung im Einheitskreis, bei der Interpretation von Kugelkoordinaten etc.).

Entscheidende Kernelemente sind:

- das rechtwinklige Dreieck als Projektionsfigur einer orthogonalen Projektion,

- die Hypotenuse als zu projizierende Seite,

- die Gegenkathete als projizierte Strecke,

- der Ausdruck $\sin (\alpha)$ als Faktor, der die Länge der zu projizierenden Strecke auf die Länge der projizierten Strecke verkürzt.

Zwar wird auch in der zweiten Klasse mathematisch das Verhältnis zweier Strecken genutzt, die Dreiecksfiguren sind jedoch in den Phänomenen nicht ,gegeben“; vielmehr geht die eine (die entsprechende Kathete) aus einer Projektion der anderen (der Hypotenuse) hervor.

\subsubsection{Formulierung konkreter Grundvorstellungen}

Es werden folgende Grundvorstellungen formuliert (für eine ausführlichere Beschreibung mit Aufgabenbeispielen s. Salle und Frohn 2017).

- Seitenverhältnisvorstellung: Sinus eines Winkels als Seitenverhältnis von Gegenkathete und Hypotenuse in einem rechtwinkligen Dreieck.

- Projektionsvorstellung: Sinus eines Winkels als Projektionsfaktor, der angibt, wie sich in einem rechtwinkligen Dreieck die Länge der Hypotenuse bei Projektion auf die durch die Gegenkathete festgelegte Gerade verringert.

Die Seitenverhältnis- und die Projektionsvorstellung lassen einen unterschiedlichen Zugang zum Einheitskreis zu. Die konsequente Verfolgung der Seitenverhältnisvorstellung führt zum Einschreiben eines rechtwinkligen Dreiecks in den Einheitskreis, während die Projektionsvorstellung zu einer Projektion des rotierenden Kreisradius auf die Koordinatenachsen führt.

In Bezug auf das Grundverständnis zum Sinus bei rechtwinkligen Dreiecken kommen die bereits beschriebenen Zusammenhänge und Unterschiede der beiden Grundvorstellungen zum Tragen. Für ausführlichere Analysen, insbesondere hinsichtlich möglicher Grundvorstellungsumbrüche, müssen zudem zwei weitere neu formulierte Grundvorstellungen - die Einheitskreis- und die Oszillationsvorstellung - berück- 
sichtigt werden, die sich auf periodische Phänomene beziehen, hier jedoch nicht explizit dargestellt werden (s. hierfür Salle und Frohn 2017).

\subsubsection{Präzisierung des Bezugsrahmens und Bewertung der didaktischen Relevanz}

Grundkenntnisse werden exemplarisch für die Anwendung der Seitenverhältnisvorstellung formuliert; sie beziehen sich beispielsweise auf die Bestandteile der Formeldarstellung $\sin (\alpha)=\frac{a}{c}$ für ein rechtwinkliges Dreieck $\triangle A B C$ :

1. $a$ und $c$ geben die Seitenlängen der Gegenkathete und der Hypotenuse des rechtwinkligen Dreiecks an.

2. $\alpha$ ist der Winkel, den Hypotenuse und Ankathete einschließen.

3. Die Hypotenuse ist die längste Seite im rechtwinkligen Dreieck, daher ist das Verhältnis $\frac{a}{c}$ immer kleiner als 1 .

4. Je länger die Gegenkathete $a$ bei gleicher Hypotenuse $c$ ist, desto größer ist $\sin (\alpha)=\frac{a}{c}$.

5. Je größer der Winkel $\alpha$, desto größer ist $\sin (\alpha)$.

6. Sind zwei Dreiecke ähnlich zueinander, so ist der Sinus der sich entsprechenden Winkel gleich.

Grundkenntnisse „als konkrete Ausprägungen von Grundvorstellungen“ (Stölting 2008, S. 39) können sich hier beispielsweise zeigen, wenn beim mentalen Variieren eines rechtwinkligen Dreiecks gefolgert wird, dass bei Beibehaltung des rechten Winkels die Hypotenuse stets größer bleibt als die Gegenkathete, das Verhältnis der beiden demnach immer zugunsten der Hypotenuse ausfällt und demnach kleiner als 1 bleibt.

Die didaktische Relevanz der formulierten Grundvorstellungen wird aktuell auf verschiedenen Ebenen überprüft (Salle und Frohn 2017, 2020).

\subsection{Grundvorstellungen zum Bruchzahlbegriff}

In der fachdidaktischen Literatur werden für den Bruchzahlbegriff drei Grundvorstellungen unterschieden: Bruch als Anteil, Bruch als Operator und Bruch als Verhältnis (Blum und Wiegand 1998; Wartha 2007). Im Folgenden werden diese Grundvorstellungen mit dem vorgestellten Verfahren rekonstruiert und hinsichtlich möglicher Ausdifferenzierungen kommentiert.

Als zentrales Konzept wird der Bruchzahlbegriff mit dem Gültigkeitsbereich der positiven rationalen Zahlen festgelegt. Dementsprechend ergibt sich ein vorläufiger Bezugsrahmen, der beispielsweise Kompetenzen zu den natürlichen Zahlen, insbesondere deren Multiplikation und Division, sowie ein kardinales Verständnis von natürlichen Zahlen enthält. Zudem greifen Kreis- oder Rechteck-Darstellungen Kompetenzen zur elementaren Geometrie auf.

Die drei Kernpunkte des zweiten Schrittes sind eine Sachanalyse, eine Analyse relevanter Phänomene und der Einbezug empirischer Ergebnisse. Zunächst können zwei zentrale Definitionen identifiziert werden: die des Bruchs und die der Bruchzahl 
(Padberg et al. 1995). Ein Bruch ist das geordnete Paar $(a, b)$, wobei $a, b \in \mathbb{N}$. Eine Bruchzahl $\frac{a}{b}$ ist die Klasse der Brüche $\left(a^{\prime}, b^{\prime}\right)$ mit $a^{\prime}, b^{\prime} \in \mathbb{N}$, d.h.:

$$
\frac{a}{b}:=\left\{\left(a^{\prime}, b^{\prime}\right) \mid a^{\prime}, b^{\prime} \in \mathbb{N} \text { mit } a \cdot b^{\prime}=a^{\prime} \cdot b\right\}
$$

Die Menge aller Bruchzahlen wird bezeichnet mit $Q^{+}$(oder auch $\mathrm{B}$ ). Alternative Definitionen für die zentralen Konzepte werden aufgrund der bestimmten Richtlinien aus Schritt 1 im Weiteren nicht berücksichtigt, weshalb auch keine Zusammenhänge zwischen verschiedenen Definitionen analysiert werden.

Hinsichtlich der Identifikation und Analyse von Anwendungen listen Padberg und Wartha (2017) eine Vielzahl von Verwendungsweisen von Brüchen auf, die in Wartha (2007) auch als „Bruchzahlaspekte“ benannt werden. Diese Aspekte ordnen und beschreiben Verwendungsweisen sowohl im alltäglichen Leben als auch in der Mathematik und strukturieren folglich die relevanten Phänomene (Padberg und Wartha 2017). Formuliert werden beispielsweise der Anteilsaspekt, der Maßzahlaspekt, der Operatoraspekt, der quasikardinale Aspekt, der Skalenwertaspekt, etc. Die Auswahl von Anwendungen und Phänomenen wird zudem vor dem Hintergrund der betrachteten mathematischen Operationen getroffen, die beim Umgang mit Bruchzahlen in den entsprechenden Kontexten relevant werden.

Die genannten Phänomene lassen sich zum Teil auch in der historischen Begriffsentwicklung nachzeichnen. Brüche wurden bereits in den frühen Hochkulturen mehrere Jahrtausende vor Christus zur Teilung von Größen, zur Bestimmung der Güte von Nahrungsmitteln im Sinne eines Anteils oder zur Angabe von Seitenverhältnissen genutzt. Systematisch wurde die Lehre der Zahlverhältnisse erstmalig bei Euklid betrachtet (Wußing 2008). Die Entwicklung der Brüche resultiert dabei ,ursprünglich aus dem Bedürfnis, das Problem des Messens auf das des Zählens zurückzuführen“ (Hefendehl-Hebeker und Schwank 2015, S. 101).

Zentrale Darstellungen beim mathematischen Arbeiten mit Brüchen sind enaktive (z.B. durchgeführte Teilungen von Gegenständen), ikonische (z.B. bildliche Darstellungen von Kreisen, Rechtecken, Strecken oder Messbechern) und symbolische Darstellungen (z.B. Bruchschreibweise, Dezimalbrüche, Divisionsausdrücke) (z. B. Malle 2004; Padberg und Wartha 2017; Schink 2013).

In zahlreichen empirischen Analysen werden vor allem typische Fehler von Schülerinnen und Schülern und die entsprechenden Ursachen in den Blick genommen (z.B. Eichelmann et al. 2012; Prediger 2011). Solche Studien zeigen insbesondere auf, welche Relevanz verschiedene Phänomene im Rahmen individueller Vorstellungen haben und wie sie dadurch die folgende Klassenbildung beeinflussen können.

Auf Basis dieser Betrachtungen wird die Klassenbildung vorgenommen.

Erste Klasse: Bruch als Anteil einer Grundmenge Die erste Klasse vereint u. a. Phänomene, in denen Brüche als Maßzahlen bei abstrakten Mengen verwendet und Anteile einzelner bzw. mehrerer konkreter Objekte beschrieben werden (Wartha 2007). Die Kernelemente sind: 
- der Nenner, der die Gesamtzahl der gleichgroßen Einheiten einer Einteilung angibt (z. B. Stücke oder Mengen),

- der Zähler, der die Zahl der gewählten Einheiten der durch den Nenner festgelegten Einteilung angibt,

- das Ganze, das die Grundmenge bzw. Maßeinheit angibt (einzelne Gegenstände, Zusammenfassungen konkreter Gegenstände oder abstrakte Größen)

- der Bruch, der mit Zähler und Nenner den Anteil am Ganzen angibt.

Zweite Klasse: Bruch als (zusammengesetzter) Operator In der zweiten Klasse werden Phänomene gegliedert, in denen der operationale Charakter eines Bruchs eine zentrale Rolle spielt: Ein Bruch wirkt hier auf Zahlen, Mengen oder Größen und verändert diese auf eine spezifische Weise (Postel 1981; Wartha 2007); hier wird auf den Prozess fokussiert, nicht auf den Anteil. Entscheidende Kernelemente sind:

- der Nenner, der angibt, in wie viele gleichgroße Teile eine Bezugsgröße geteilt wird (Divisionsoperator)

- der Zähler, der angibt, wie oft eine Bezugsgröße vervielfacht wird (Multiplikationsoperator),

- die Bezugsgröße, auf die die Operatoren bzw. der zusammengesetzte Operator wirkt,

- der Bruch, der den zusammengesetzten Operator darstellt.

Dritte Klasse: Bruch als Verhältnis von Zahlen und Größen Eine dritte Klasse schließt Phänomene zusammen, in denen Brüche Verhältnisse darstellen. Hier ist ein Bruch ein Vergleichsindex, eine ,ratio number“ (Kieren 1976, S. 111) mit oder ohne Dimension (Wartha 2007). Entscheidende Kernelemente sind:

- der Nenner, der eine Größe (oder Zahl) angibt,

- der Zähler, der eine Größe (dieselbe wie im Nenner oder eine andere) angibt,

- der Bruch, der das Verhältnis zwischen den beiden Verglichenen quantifiziert.

Im Rahmen der Herleitung werden von den Forschenden Entscheidungen getroffen, die beispielsweise die Auswahl einbezogener Definitionen und Phänomene oder die Rolle empirischer Ergebnisse betreffen. Im Folgenden wird exemplarisch aufgezeigt, wie abweichende Entscheidungen zu ausdifferenzierteren Grundvorstellungen führen können.

- Erweiterte Auswahl der einbezogenen Phänomene: In der durchgeführten Herleitung wurden mit Blick auf den Bezugsrahmen und den von Anwendungen geprägten Sekundarstufenunterricht kaum Phänomene berücksichtigt, die bei einer Thematisierung von Brüchen als Äquivalenzklassen eine Rolle spielen (Wartha 2007). Nimmt man solche Phänomene hinzu, die sich auf den Umgang mit mathematischen Strukturen und Relationen beziehen, könnte dies zu einer weiteren Klasse „Bruch als Äquivalenzklasse“ führen, was zudem die obige mathematische Definition der Bruchzahl aufgreifen würde.

- Mögliche Ausdifferenzierung der Klasse „Bruch als Anteil“: Auf der mathematisch-sachanalytischen Ebene ließe sich die Anteilsbildung an mehreren Ganzen 
als eigene Klasse von den Phänomenen der Anteilsbildung an einem Ganzen abgrenzen (z.B. Schink 2013). Dies würde durch empirische Belege gestützt, da Schülerinnen und Schüler im ersten Fall deutlich mehr Schwierigkeiten zeigen (z.B. Padberg und Krüger 1997).

Auf Basis der ausgeführten Klassen können nun die drei Grundvorstellungen formuliert werden, die als „Bruch als Anteil“", „Bruch als Operator“ und „Bruch als Verhältnis“ ausführlich bei Wartha (2007) beschrieben werden.

\section{Perspektiven}

Im vorliegenden Beitrag wird ein Verfahrensrahmen für die Herleitung von Grundvorstellungen als normative Leitlinien vorgeschlagen. Die vorgestellten Schritte stellen keinen rigiden Algorithmus dar: Der Gefahr einer Algorithmisierung wird begegnet, indem immer wieder zu vorigen Schritten zurückgegangen werden kann und damit ein flexibles Gerüst zur Herleitung von Grundvorstellungen beschrieben wird. Innerhalb dieses Gerüsts müssen Spezifika einzelner mathematischer Begriffe stets bedacht und konkretisiert werden. Die vorgestellten Schritte sind aus unserer Sicht zentraler Kern jedweder Analysen, die die Neu-Formulierung oder das Nachvollziehen konkreter Grundvorstellungen zum Ziel haben.

Die Ausführungen sollen zum Ausdruck bringen, dass Grundvorstellungen Kategorien sind, die von Forscherinnen und Forschern konstruiert werden. Sie basieren zwar auf mathematischen und phänomenologischen Grundlagen, sind in ihrer Formulierung jedoch auf Basis unterschiedlich gewählter Bezugsrahmen, Gültigkeitsbereiche, Definitionen und Phänomene immer auch durch Entscheidungen der Forschenden geprägt; alternative Entscheidungen können daher zu abweichenden Konkretisierungen und Ausdifferenzierungen von Grundvorstellungen führen. Jedoch ermöglicht erst die Explikation solcher Entscheidungen und des generellen Vorgehens eine Diskussion der formulierten Leitlinien und ihrer Entstehung. Wenn auch eine Darstellung aller konkret durchgeführten Schritte - gerade in Praxisbeiträgen - oftmals nicht möglich ist, gibt ein grundlegender Rahmen auch in kürzeren Darstellungen eine Orientierung, um zentrale Entscheidungen zu präzisieren und so die Nachvollziehbarkeit zu gewährleisten.

Neben den grundlegenden Schritten 1-4 stellen die in Schritt 5 beschriebenen Bewährungsebenen wichtige Mittel zur Weiterentwicklung der hergeleiteten Grundvorstellungen dar. Indem neu formulierte Grundvorstellungen beispielsweise Gegenstand empirischer Studien werden, kann ihre didaktische Relevanz überprüft werden. Obgleich in diesem Spannungsfeld bereits einige didaktisch gewinnbringende Arbeiten vorliegen, sind insbesondere die theoretische Auskleidung (vgl. Reinhold 2019) und die Anbindung der deskriptiven Ebene an die normativen Überlegungen sowie die damit zusammenhängende Verankerung des Grundvorstellungsbegriffs in kognitionspsychologischen Grundlagentheorien Desiderata für weitere Forschungsarbeiten (vgl. Hanke und Schäfer 2017; Schacht 2012; Vogel 2016). Weiterhin können Impulse aus der Unterrichtspraxis zur Ausdifferenzierung bestehender Grundvorstellungen anregen. 
Der vorgestellte Verfahrensrahmen leistet einen Beitrag zur Weiterentwicklung der Grundvorstellungsidee: Wir hoffen, dass durch die Systematisierung der Herleitung mathematisch-inhaltlicher Leitlinien die produktive Verschränkung normativer Analysen, deskriptiver Untersuchungen und konstruktiver Lehrgangsgestaltung im Sinne eines vielseitigen und praxisrelevanten Grundvorstellungskonzeptes intensiviert werden kann.

Funding Open Access funding enabled and organized by Projekt DEAL.

Open Access Dieser Artikel wird unter der Creative Commons Namensnennung 4.0 International Lizenz veröffentlicht, welche die Nutzung, Vervielfältigung, Bearbeitung, Verbreitung und Wiedergabe in jeglichem Medium und Format erlaubt, sofern Sie den/die ursprünglichen Autor(en) und die Quelle ordnungsgemäß nennen, einen Link zur Creative Commons Lizenz beifügen und angeben, ob Änderungen vorgenommen wurden.

Die in diesem Artikel enthaltenen Bilder und sonstiges Drittmaterial unterliegen ebenfalls der genannten Creative Commons Lizenz, sofern sich aus der Abbildungslegende nichts anderes ergibt. Sofern das betreffende Material nicht unter der genannten Creative Commons Lizenz steht und die betreffende Handlung nicht nach gesetzlichen Vorschriften erlaubt ist, ist für die oben aufgeführten Weiterverwendungen des Materials die Einwilligung des jeweiligen Rechteinhabers einzuholen.

Weitere Details zur Lizenz entnehmen Sie bitte der Lizenzinformation auf http://creativecommons.org/ licenses/by/4.0/deed.de.

\section{Literatur}

Bauersfeld, H. (1983). Subjektive Erfahrungsbereiche als Grundlage einer Interaktionstheorie des Mathematiklernens und -lehrens. In H. Bauersfeld, H. Bussmann, G. Krummheuer, J. H. Lorenz \& J. Voigt (Hrsg.), Lernen und Lehren von Mathematik (S. 1-56). Köln: Aulis.

Bender, P. (1991). Ausbildung von Grundvorstellungen und Grundverständnissen - ein tragendes didaktisches Konzept für den Mathematikunterricht - erläutert an Beispielen aus den Sekundarstufen. In H. Postel, A. Kirsch \& W. Blum (Hrsg.), Mathematik lehren und lernen: Festschrift für Heinz, Griesel (S. 48-60). Hannover: Schroedel.

Blackett, N., \& Tall, D. (1991). Gender and the versatile learning of trigonometry using computer software. In F. Furinghetti (Hrsg.), Proceedings of the 15th conference of the International Group for the Psychology of Mathematics Education (S. 144-151). Assisi: PME.

Blum, W., \& Kirsch, A. (1979). Zur Konzeption des Analysisunterrichts in Grundkursen. Der Mathematikunterricht, 25(3), 6-24.

Blum, W., \& Wiegand, B. (1998). Wie kommen die deutschen TIMSS-Ergebnisse zustande? Ein Interpretationsansatz auf der Basis stoffdidaktischer Analysen. In W. Blum \& M. Neubrand (Hrsg.), TIMSS und der Mathematikunterricht (S. 28-34). Hannover: Schroedel.

Brown, S. A. (2005). The trigonometric connection: students' understanding of sine and cosine. Dissertation, Illinois State University.

Bruner, J.S. (1964). The course of cognitive growth. American Psychologist, 19(1), 1-15.

Dörfler, W. (1990). Prototypen und Protokolle als kognitive Mittel gegen Bedeutungslosigkeit und Entfremdung im Mathematikunterricht. In Landesinstitut für Schule und Weiterbildung (Hrsg.), Die Zukunft des Mathematikunterrichts (S. 102-109). Soest: Landesinstitut für Schule und Weiterbildung.

Duit, R., \& Treagust, D. F. (2003). Conceptual change: a powerful framework for improving science teaching and learning. International Journal of Science Education, 25(6), 671-688.

Eichelmann, A., Narciss, S., Schnaubert, L., \& Melis, E. (2012). Typische Fehler bei der Addition und Subtraktion von Brüchen - Ein Review zu empirischen Fehleranalysen. Journal für MathematikDidaktik, 33(1), 29-57.

Etzold, H. (2019). Grundvorstellungen ausbilden - Digitale Medien als Lernmodelle nutzen. In A. Frank, S. Krauss \& K. Binder (Hrsg.), Beiträge zum Mathematikunterricht (S. 221-224). Münster: WTM. 
Filler, A. (2006). Einbeziehung von Elementen der 3D-Computergrafik in den Mathematikunterricht der Sekundarstufe II im Stoffgebiet Analytische Geometrie. Habilitationsschrift. Berlin: Humboldt-Universität.

Filler, A. (2018). Trigonometrie. In H.-G. Weigand, A. Filler, R. Hölzl, S. Kuntze, M. Ludwig, J. Roth \& G. Wittmann, et al. (Hrsg.), Didaktik der Geometrie für die Sekundarstufe I (S. 227-253). Berlin: Springer.

Fischbein, E. (1987). Intuition in science and mathematics: an educational approach. Dordrecht: Reidel.

Freudenthal, H. (1983). Didactical phenomenology of mathematical structures. Dordrecht: Reidel.

Frohn, D. (2020). Mehr als Orthogonalität. Das Skalarprodukt beziehungsreich anwenden - mit Grundvorstellungen. mathematik lehren, 218, 33-38.

Frohn, D., \& Salle, A. (Hrsg.). (2017). Periodische Vorgänge. mathematik lehren, Bd. 204.

Funk, S. (2013). sin, cos, tan ... Wie bitte? Praxis der Mathematik, 54/55, 27-29.

Gallin, P., \& Ruf, U. (1993). Sprache und Mathematik in der Schule. Ein Bericht aus der Praxis. Journal für Mathematik-Didaktik, 14, 3-33.

Greefrath, G., Oldenburg, R., Siller, H.-S., Ulm, V., \& Weigand, H.-G. (2016a). Didaktik der Analysis: Aspekte und Grundvorstellungen zentraler Begriffe. Berlin, Heidelberg: Springer.

Greefrath, G., Oldenburg, R., Siller, H.-S., Ulm, V., \& Weigand, H.-G. (2016b). Aspects and "Grundvorstellungen" of the concepts of derivative and integral: subject matter-related didactical perspectives of concept formation. Journal für Mathematik-Didaktik, 37(S1), 99-129.

Griesel, H. (1970). Der wissenschaftliche Hintergrund der Bruchrechnung. Der Mathematikunterricht, $16(2), 5-29$.

Griesel, H. (1971). Die mathematische Analyse als Forschungsmittel in der Didaktik der Mathematik. In Beiträge zum Mathematikunterricht 1971 (S. 72-81). Hannover: Schroedel.

Griesel, H. (2016). Die Vergleichstheorie des Messens und ihre Anwendung in der mathematikdidaktischen Grundlagenforschung. Journal für Mathematik-Didaktik, 37(1), 5-30.

Griesel, H., vom Hofe, R., \& Blum, W. (2019). Das Konzept der Grundvorstellungen im Rahmen der mathematischen und kognitionspsychologischen Begrifflichkeit in der Mathematikdidaktik. Journal für Mathematik-Didaktik, 40(1), 123-133.

Hafner, T. (2012). Proportionalität und Prozentrechnung in der Sekundarstufe I: Empirische Untersuchung und didaktische Analysen. Wiesbaden: Vieweg \& Teubner.

Hanke, E., \& Schäfer, I. (2017). Students' view of continuity: an empirical analysis of mental images and their usage. In T. Dooley \& G. Gueudet (Hrsg.), Proceedings of CERME 10 (S. 2081-2088). Dublin: DCU Institute of Education and ERME.

Hasemann, K., \& Gasteiger, H. (2014). Anfangsunterricht Mathematik. Berlin, Heidelberg: Springer.

Hefendehl-Hebeker, L., \& Schwank, I. (2015). Arithmetik: Leitidee Zahl. In R. Bruder, L. Hefendehl-Hebeker, B. Schmidt-Thieme \& H.-G. Weigand (Hrsg.), Handbuch der Mathematikdidaktik (S. 77-115). Berlin, Heidelberg: Springer.

vom Hofe, R. (1995). Grundvorstellungen mathematischer Inhalte. Heidelberg: Spektrum.

vom Hofe, R. (2003a). Grundbildung durch Grundvorstellungen. mathematik lehren, 118, 4-8.

vom Hofe, R. (Hrsg.). (2003b). Grundvorstellungen entwickeln. mathematik lehren, Bd. 118.

vom Hofe, R., \& Blum, W. (2016). "Grundvorstellungen" as a category of subject-matter didactics. Journal für Mathematik-Didaktik, 37(S1), 225-254.

vom Hofe, R., Humpert, B., Griesel, H., \& Postel, H. (Hrsg.). (2014). Schülerband Nordrhein-Westfalen, Gemeinschaftsschule, Realschule, Sekundarschule, Verbundschule. Mathematik heute, Bd. 7. Braunschweig: Schroedel.

vom Hofe, R., Kleine, M., Blum, W., \& Pekrun, R. (2005). Zur Entwicklung mathematischer Grundbildung in der Sekundarstufe I - theoretische, empirische und diagnostische Aspekte. In M. Hasselhorn, H. Marx \& W. Schneider (Hrsg.), Diagnostik von Mathematikleistungen (S. 263-292). Göttingen: Hogrefe.

Holland, G. (1974). Die Bedeutung von Konstruktionsaufgaben für den Geometrieunterricht. Der Mathematikunterricht, 20(1), 71-86.

Hußmann, S., \& Prediger, S. (2016). Specifying and structuring mathematical topics: a four-level approach for combining formal, semantic, concrete, and empirical levels exemplified for exponential growth. Journal für Mathematik-Didaktik, 37(S1), 33-67.

Juter, K. (2010). Conceptual change and connections in analysis. In V. Durand-Guerrier, S. Soury-Lavergne \& F. Arzarello (Hrsg.), Proceedings of CERME 6 (S. 2276-2285). Lyon: Institut National de Recherche Pédagogique and ERME. 
Kendal, M., \& Stacey, K. (1996). Trigonometry: comparing ratio and unit circle methods. In P. Clarkson (Hrsg.), Technology in Mathematics Education-Proceedings of the 19th Annual Conference of the Mathematics education Research group of Australasia (S. 322-329). Melbourne: MERGA.

Kieren, T.E. (1976). On the mathematical, cognitive and instructional foundations of rational numbers. In R. Lesh (Hrsg.), Number and measurement (S. 101-144). Columbus: Smeac.

Kirsch, A. (1987). Mathematik wirklich verstehen. Köln: Aulis.

Kirsch, A. (2000). Aspects of simplification in mathematics teaching. In I. Westbury, S. Hopmann \& K. Riquarts (Hrsg.), Teaching as a reflective practice - the German didactic tradition (S. 267-284). Mahwah: Lawrence Erlbaum.

Kleine, M., Jordan, A., \& Harvey, E. (2005). With a focus on 'Grundvorstellungen' part 1: a theoretical integration into current concepts. Zentralblatt für Didaktik der Mathematik, 37(3), 226-233.

Klinger, M. (2019). Grundvorstellungen versus Concept Image? Gemeinsamkeiten und Unterschiede beider Theorien am Beispiel des Funktionsbegriffs. In A. Büchter, M. Glade, R. Herold-Blasius, M. Klinger, F. Schacht \& P. Scherer (Hrsg.), Vielfältige Zugänge zum Mathematikunterricht (S. 61-75). Wiesbaden: Springer.

Leuders, T., Hußmann, S., Barzel, B., \& Prediger, S. (2011). „Das macht Sinn!“ Sinnstiftung mit Kontexten und Kernideen. Praxis der Mathematik in der Schule, 37, 2-9.

Lorenz, J.H. (2017). Einige Anmerkungen zur Repräsentation von Wissen über Zahlen. Journal für Mathematik-Didaktik, 38(1), 125-139.

Malle, G. (2000). Zwei Aspekte von Funktionen: Zuordnung und Kovariation. mathematik lehren, 103, 4-7.

Malle, G. (2001). Genetisch in die Trigonometrie. mathematik lehren, 109, 40-44.

Malle, G. (2003). Vorstellungen vom Differenzenquotienten fördern. mathematik lehren, 118, 57-62.

Malle, G. (2004). Grundvorstellungen zu Bruchzahlen. mathematik lehren, 123, 4-8.

Malle, G., \& Malle, S. (2003). Was soll man sich unter einer Wahrscheinlichkeit vorstellen? mathematik lehren, 118, 52-56.

Merzbach, U.C., \& Boyer, C. B. (2011). A history of mathematics. Hoboken: John Wiley.

Müller, M. (1997). Analyse von Schülerfehlern in der Trigonometrie: Ursachenforschung, Diagnose und Fehlerkorrektur anhand von Schularbeitsheften, Interviews und Übungen nach dem Prinzip des Mastery-Learning. Dissertation, Grund- und Integrativwissenschaftliche Fakultät der Universität Wien.

Niedersächsisches Kultusministerium (2018). Kerncurriculum für das Gymnasium - gymnasiale Oberstufe, die Gesamtschule - gymnasiale Oberstufe das Berufliche Gymnasium, das Abendgymnasium, das Kolleg. Hannover: Unidruck.

Padberg, F., \& Krüger, F. (1997). Ordnen von Brüchen - Lösungsstrategien und typische Fehler. Mathematische Unterrichtspraxis, 18(2), 35-41.

Padberg, F., \& Wartha, S. (2017). Didaktik der Bruchrechnung. Berlin: Springer.

Padberg, F., Danckwerts, R., \& Stein, M. (1995). Zahlbereiche. Heidelberg, Berlin: Spektrum.

Postel, H. (1981). Größen- oder Operatorkonzept in der Bruchrechnung? Der Mathematikunterricht, 27(4), $16-46$.

Prediger, S. (2008). The relevance of didactic categories for analysing obstacles in conceptual change: revisiting the case of multiplication of fractions. Learning and Instruction, 18(1), 3-17.

Prediger, S. (2010). „Aber wie sag ich es mathematisch?“ - Empirische Befunde und Konsequenzen zum Lernen von Mathematik als Mittel zur Beschreibung von Welt. In D. Höttecke (Hrsg.), Entwicklung naturwissenschaftlichen Denkens zwischen Phänomen und Systematik. Jahrestagung der Gesellschaft für Didaktik der Chemie und Physik in Dresden 2009 (S. 6-20). Berlin: LIT.

Prediger, S. (2011). Why Johnny can't apply multiplication? Revisiting the choice of operations with fractions. International Electronic Journal of Mathematics Education, 6(2), 65-88.

Prediger, S., Gravemeijer, K., \& Confrey, J. (2015). Design research with a focus on learning processes-an overview on achievements and challenges. Zentralblatt für Didaktik der Mathematik, 47(6), 877-891.

Reinhold, F. (2019). Wirksamkeit von Tablet-PCs bei der Entwicklung des Bruchzahlbegriffs aus mathematikdidaktischer und psychologischer Perspektive: Eine empirische Studie in Jahrgangsstufe 6. Wiesbaden: Springer.

Rogers, L., \& Pope, S. (2016). Working group report: a brief history of trigonometry for mathematics educators. In G. Adams (Hrsg.), Proceedings of the British Society for Research into Learning Mathematics (Bd. 36, S. 103-108). Manchester: Metropolitan University.

Roos, A.-K. (2020). Mathematisches Begriffsverständnis im Übergang Schule-Universität: Verständnisschwierigkeiten von Mathematik an der Hochschule am Beispiel des Extrempunktbegriffs. Wiesbaden: Springer.

Roth, J. (2005). Bewegliches Denken im Mathematikunterricht. Hildesheim: Franzbecker. 
Ruf, U. (2008). Das dialogische Lernmodell. In U. Ruf, S. Keller \& F. Winter (Hrsg.), Besser lernen im Dialog: Dialogisches Lernen in der Unterrichtspraxis (S. 13-23). Seelze-Velber: Klett, Kallmeyer.

Salle, A., \& Frohn, D. (2017). Grundvorstellungen zu Sinus und Cosinus. mathematik lehren, 204, 8-12.

Salle, A., \& Frohn, D. (2020). Transferprozesse am Einheitskreis - Alternative Sinus- und Kosinusfunktionen. mathematik lehren, 218, 27-32.

Schacht, F. (2012). Mathematische Begriffsbildung zwischen Implizitem und Explizitem: Individuelle Begriffsbildungsprozesse zum Muster- und Variablenbegriff. Wiesbaden: Vieweg + Teubner.

Schecker, H., \& Duit, R. (2018). Schülervorstellungen und Physiklernen. In H. Schecker, T. Wilhelm, M. Hopf \& R. Duit (Hrsg.), Schülervorstellungen und Physikunterricht: Ein Lehrbuch für Studium, Referendariat und Unterrichtspraxis (S. 1-21). Berlin: Springer.

Schink, A. (2013). Flexibler Umgang mit Brüchen: Empirische Erhebung individueller Strukturierungen zu Teil, Anteil und Ganzem. Wiesbaden: Springer.

Stachowiak, H. (1973). Allgemeine Modelltheorie. Wien: Springer.

Städtler, T. (2003). Lexikon der Psychologie: Wörterbuch, Handbuch, Studienbuch. Stuttgart: Kröner.

Ständige Konferenz der Kultusminister der Länder in der Bundesrepublik Deutschland (Hrsg.). (2015). Bildungsstandards im Fach Mathematik für die Allgemeine Hochschulreife: Beschluss der Kultusministerkonferenz vom 18.10.2012. Köln: Link.

Stölting, P. (2008). Die Entwicklung funktionalen Denkens in der Sekundarstufe I - vergleichende Analysen und empirische Studien zum Mathematikunterricht in Deutschland und Frankreich. Dissertation, Universität Regensburg, Paris Diderot.

Tall, D., \& Vinner, S. (1981). Concept image and concept definition in mathematics with particular reference to limits and continuity. Educational Studies in Mathematics, 12(2), 151-169.

Thompson, P. W. (2008). Conceptual analysis of mathematical ideas: Some spadework at the foundations of mathematics education. In O. Figueras, J. L. Cortina, S. Alatorre, T. Rojano \& A. Sepulveda (Hrsg.), Proceedings of the Annual Meeting of the International Group for the Psychology of Mathematics Education (Bd. 1, S. 31-49). Morelia: PME.

Vinner, S., \& Hershkowitz, R. (1980). Concept images and common cognitive paths on the development of some simple geometrical concepts. In R. Karplus (Hrsg.), Proceedings of the Fourth International Conference for the Psychology of Mathematics Education (S. 177-184). Berkeley: Lawrence Hall of Science.

Vogel, M. (2016). Mentale Modelle - Ausgewählte Aspekte mathematikdidaktischer Adaptionen. In Institut für Mathematik und Informatik (Hrsg.), Beiträge zum Mathematikunterricht 2016. Münster: WTM.

Vohns, A. (2005). Fundamentale Ideen und Grundvorstellungen: Versuch einer konstruktiven Zusammenführung am Beispiel der Addition von Brüchen. Journal für Mathematik-Didaktik, 26(1), 52-79.

Vollrath, H.-J. (1987). Didaktische Phänomenologie als Grundlage für die Erforschung der Konstitution mentaler Objekte - Gedanken zu Freudenthals Buch. Journal für Mathematik-Didaktik, 8(4), 247-256.

Vollrath, H.-J. (1989). Funktionales Denken. Journal für Mathematik-Didaktik, 10(1), 3-37.

Vosniadou, S., \& Verschaffel, L. (2004). Extending the conceptual change approach to mathematics learning and teaching. Learning and Instruction, 14(5), 445-451.

Wartha, S. (2007). Längsschnittliche Untersuchungen zur Entwicklung des Bruchzahlbegriffs. Hildesheim: Franzbecker.

Wartha, S., \& Schulz, A. (2018). Rechenproblemen vorbeugen. Berlin: Cornelsen.

Weber, C. (2013). Grundvorstellungen zum Logarithmus - Bausteine für einen verständlichen Unterricht. In H. Allmendinger, K. Lengnink, A. Vohns \& G. Wickel (Hrsg.), Mathematik verständlich unterrichten (S. 79-98). Wiesbaden: Springer.

Weber, C. (2016). Making logarithms accessible_-Operational and structural basic models for logarithms. Journal für Mathematik-Didaktik, 37(S1), 69-98.

Weigand, H.-G. (2015). Begriffsbildung. In R. Bruder, L. Hefendehl-Hebeker, B. Schmidt-Thieme \& H.G. Weigand (Hrsg.), Handbuch der Mathematikdidaktik (S. 255-278). Berlin, Heidelberg: Springer.

Wessel, J. (2015). Grundvorstellungen und Vorgehensweisen bei der Subtraktion: Stoffdidaktische Analysen und empirische Befunde von Schülerinnen und Schülern des 1. Schuljahres. Wiesbaden: Springer.

Wußing, H. (2008). 6000 Jahre Mathematik: Eine kulturgeschichtliche Zeitreise - 1. Von den Anfängen bis Leibniz und Newton. Berlin, Heidelberg: Springer. 